\title{
Treatment Patterns and Clinical Outcomes in Elderly Patients with Breast Cancer
}

\author{
Kyu Min Kang, M.D. ${ }^{1 *}$, Eun-Kyu Kim, M.D., Ph.D. ${ }^{*}$, Hyunju Kim, M.D. ${ }^{1}$, Jee Hyun Kim, M.D., Ph.D. ${ }^{2}$, \\ Se Hyun Kim, M.D. ${ }^{2}$, Koung Jin Suh, M.D. ${ }^{2}$, In Ah Kim, M.D., Ph.D. ${ }^{3}$, Sumin Chae, M.D. ${ }^{4}$, \\ Sung-Won Kim, M.D., Ph.D. ${ }^{5}$, Eunyoung Kang, M.D., Ph.D. ${ }^{1}$ \\ ${ }^{1}$ Department of Surgery, ${ }^{2}$ Department of Internal Medicine, ${ }^{3}$ Department of Radiation Oncology, Seoul National University College of Medicine, Seoul \\ National University Bundang Hospital, Seongnam; ${ }^{4}$ Department of Surgery, Kyung Hee University School of Medicine, Kyung Hee University Medical \\ Center, Seoul; ${ }^{5}$ Department of Surgery, Daerim St. Mary's Hospital, Seoul, Korea
}

Purpose: Although the number of elderly patients with breast cancer is increasing as the population ages, their treatment is controversial. We evaluated the prognostic factors associated with survival in elderly breast cancer patients and assessed the impact of comorbidity on prognosis. Methods: This study included 362 patients (aged $\geq 65$ years) who underwent surgery for breast cancer in our institution between 2003 and 2014. The patients were divided into early-aged (65-74 years) and late-aged ( $\geq 75$ years) groups. Comorbidity was parametrized using the Charlson comorbidity index (CCl). Kaplan-Meier analysis was used to analyze overall survival (OS) and distant metastasis-free survival (DMFS). Prognostic factors were evaluated by Cox proportional hazards regression. Results: The surgical method, subtypes, stage, and oncological features were similar between early- and late-aged groups; however, smaller proportions of patients in the late-aged group received chemotherapy ( $12.9 \%$ vs. $45.5 \%)$ and endocrine therapy (55.3\% vs. $73.3 \%$ ). In multivariable analysis, the poor prognostic factors associated with DMFS and OS were high $\mathrm{CCl}$, high histologic grade, and advanced stage. Chemotherapy, endocrine therapy, and radiotherapy were not significantly related to DMFS and OS. Conclusion: In this study, adjuvant treatments did not affect the prognosis of elderly patients with breast cancer. To clarify the effects of adjuvant therapies in these patients, a large-scale retrospective study that considers not only tumor characteristics but also life expectancy is necessary.

Key Words: Aged, Breast neoplasms, Comorbidity, Prognosis

\section{INTRODUCTION}

The World Health Organization criteria categorize individuals aged 65 years and older as 'elderly' [1]. According to the Korea Statistical Office, elderly people will account for $28.7 \%$ of the Korean population by 2035 [2]. Moreover, the life expectancy of Korean women born in 2030 is projected to be 86.7 years, which is the longest in the world [3]. As the average life expectancy increases, the proportion of elderly patients with breast cancer is also projected to rise. Despite the expected increase in elderly breast cancer patients, there is insufficient evidence regarding the most suitable treatment for these women.

Correspondence: Eunyoung Kang, M.D., Ph.D.

Department of Surgery, Seoul National University College of Medicine, Seoul National University Bundang Hospital, 82 Gumi-ro 173beon-gil, Bundang-gu, Seongnam 13620, Korea

Tel: +82-31-787-7102, Fax:+82-31-787-4078, E-mail: key5002@gmail.com

*These authors contributed equally to this work.

Received: Nov 19, 2019 Revised: Jan 13, 2020 Accepted: Feb 18, 2020
It is difficult to determine suitable therapeutic plans in these patients because aging is a complex and heterogeneous process in which chronological age does not always reflect the physiological state. A variety of factors should be considered when treating geriatric patients, including comorbidities, life expectancy, cognitive ability, performance status, concurrent medication, and socioeconomic support $[4,5]$. Elderly breast cancer patients tend to be undertreated for the reasons mentioned above and the survival rate of elderly patients who are undertreated is lower than that of those who receive adjuvant systemic therapy [6-9]. Moreover, the prognostic factors associated with older patients with breast cancer may include not only age but also comorbidities. Elderly breast cancer patients with multiple comorbidities have high rates of mortality and complications associated with adjuvant therapy [10-14].

Therefore, it is necessary to understand how elderly patients with breast cancer are treated and to improve the quality of current care. This study investigated the patterns of treatment and prognostic fac- 
tors associated with survival in elderly patients with breast cancer.

\section{METHODS}

\section{Patients and data collection}

We retrospectively reviewed the data of 560 patients over 65 years of age who underwent surgery for breast cancer at the Seoul National University Bundang Hospital between 2003 and 2014. Patients with stage IV cancer $(n=17)$, coexisting cancers of other organs $(n=53)$, ductal carcinoma in situ $(\mathrm{n}=97)$, and solid neuroendocrine cancer or malignant adenomyoepithelioma $(n=16)$ were excluded. A total of 362 patients were included in the analysis. Figure 1 shows a diagram of the patient selection process. The Institutional Review Board of Seoul National University Bundang Hospital approved this study (IRB No. B-1708/412-135). The requirement for informed consent was waived due to the retrospective study design.

Data on the type of surgery in the breast and axilla; comorbidities; histologic type; histologic grade (HG); stage; estrogen receptor (ER), progesterone receptor (PR), and human epidermal growth factor receptor 2 (HER2) expression; adjuvant therapy (chemotherapy, radiotherapy, hormone therapy, and targeted therapy); and disease status at the last follow-up visit were collected from electronic medical records (EMRs). Data on survival status were collected in conjunction with the Ministry of the Interior and Safety. Our study classified comor-

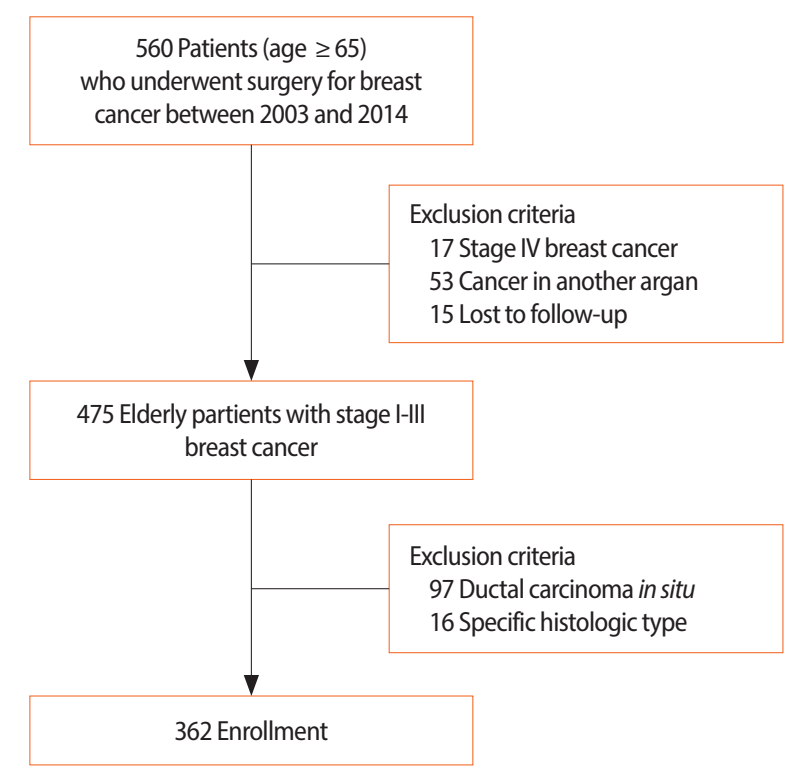

Figure 1. Study population selection. bidities into four categories ( $0,1,2$, and $\geq 3)$ using the Charlson comorbidity index (CCI), which is composed of 19 disease groups assigning scores of $1,2,3$, and 6 to each of a patient's comorbid conditions [15]. Histologic type was divided into favorable (tubular carcinoma and mucinous carcinoma) and usual (invasive ductal carcinoma, invasive lobular carcinoma, metaplastic carcinoma, and mixed ductal \& lobular carcinoma) types. The staging system was based on the American Joint Committee on Cancer 7th edition [16]. Breast cancer subtypes were classified as luminal type A, luminal type B, HER2-enriched, and triple-negative breast cancer (TNBC) on based on ER, PR, HER2, and Ki-67 expression, as suggested by the 2013 St. Gallen Consensus [17]. Samples were defined as hormone receptor-positive when at least $1 \%$ of cells were positive by immunohistochemistry. The Ki-67 index was divided into high $(\geq 14 \%)$ and low $(<14 \%)$ expression. The adjuvant chemotherapy regimens administered included doxorubicin and cyclophosphamide (AC); AC plus docetaxel; epirubicin and cyclophosphamide; cyclophosphamide, methotrexate, and fluorouracil (CMF); fluorouracil, doxorubicin, and cyclophosphamide; and cyclophosphamide, epirubicin, and fluorouracil.

\section{Statistical analysis}

The patients were divided into early-aged $(65-74$ years, $n=277)$ and late-aged ( $\geq 75$ years, $n=85$ ) groups. The differences between the early-aged and late-aged groups were analyzed using the Chi-square or Fisher's exact tests. Overall survival (OS) and distant metastasis-free survival (DMFS) were estimated using Kaplan-Meier analysis and log-rank tests were used to compare survival differences according to patient characteristics. Distant metastasis included recurrence in the contralateral breast, cervical lymph nodes (level I-V), contralateral lymph nodes (axillary, internal mammary, supraclavicular, and infraclavicular lymph nodes), bone, and visceral organs (lung, liver, and other organs) [18]. Follow-up was completed on July 31, 2017. OS was defined as the time interval from the date of breast cancer surgery to the date of death from any cause or the last follow-up date. DMFS was defined as the time interval from the date of breast cancer surgery to the date of distant metastasis. Univariable and multivariable Cox proportional hazards regression analyses were used to evaluate prognostic factors. All analyses were performed using IBM SPSS Statistics for Windows, version 22.0 (IBM Corp., Armonk, USA). Statistical significance was defined as $p<0.05$. 


\section{RESULTS}

\section{Patient characteristics}

We analyzed 362 elderly patients with stage I-III breast cancer. Their mean age was 71.1 years (range $65-88$ years). We compared clinicopathological characteristics between the early-aged and lateaged groups (Table 1). The surgery types for the breast and axilla, histologic type, HG, stage, subtype, and target therapy (trastuzumab) were similar between groups. The proportion of patients with CCI $\geq$ 3 was higher in the late-aged group than that in the early-aged group (37.6\% vs. $16.6 \%, p<0.001)$. Although no difference in stage distribution was observed between the two groups, significantly higher proportions of patients in the early-aged group received chemotherapy (45.5\% vs. $12.9 \%, p<0.001)$ and radiotherapy (63.9\% vs. $43.5 \%$, $p<0.001)$. Even when analyzed by stage, the proportion of patients who underwent chemotherapy was significantly higher in the early-aged group than that in the late-aged group for each stage (Table 2).

\section{Clinical outcomes}

The median follow-up period was 76.7 months (range 30-165 months). Distant metastasis was detected in 14 (5.1\%) and eight (9.4\%) patients in the early-aged and late-aged groups, respectively. The sites of distant metastasis were the lung $(n=7)$, liver $(n=5)$, bone $(n=4)$, and contralateral breast $(n=3)$ and three patients had multiple metastases. Twenty-four and 13 patients in the early-aged and late-aged groups died, respectively. EMR review revealed two patients with chemotherapy-related deaths (pancytopenia and cardiac toxicity, respectively), eight patients with deaths due to breast cancer progression, and 11 patients with deaths from other causes (two from brain infarction; two from gastric ulcer bleeding; and one each from gastric angiodysplasia, Parkinson disease, biliary sepsis, primary biliary cirrhosis, superior vena cava syndrome, lymphoma, and angina). We could not confirm the cause of death in 16 patients (43\%) due to loss from follow-up. In the entire cohort, the 5-year DMFS and OS were 96.2\% and 93.5\%, respectively. Survival by age, HG, stage, CCI, and subtype are shown in Figure 2. The OS was lower in patients with a higher HG (96.3\% for grade 1 vs. $87.1 \%$ for grade 3, $p=0.004)$, higher stage $(96.0 \%$ for stage I vs. $80.9 \%$ for stage III, $p<0.001$ ), and higher CCI score (96.1\% for CCI 0 vs. $84.1 \%$ for CCI $3, p<0.001$ ). Similar results were observed for DMFS. Regarding subtype, OS and DMFS were lowest
Table 1. Patient clinicopathological characteristics

\begin{tabular}{|c|c|c|c|}
\hline Characteristic & $\begin{array}{c}\text { Age } 65-74 \\
(n=277) \\
\text { No. }(\%)\end{array}$ & $\begin{array}{c}\text { Age } \geq 75 \\
(n=85) \\
\text { No. }(\%)\end{array}$ & $p$-value* \\
\hline \multicolumn{4}{|l|}{ Breast operation type } \\
\hline Total mastectomy & $97(35.0)$ & $38(44.7)$ & 0.069 \\
\hline Breast conserving surgery & $180(65.0)$ & $47(55.3)$ & \\
\hline \multicolumn{4}{|l|}{ Axilla operation type } \\
\hline Sentinel lymph node biopsy & $196(70.8)$ & $62(72.9)$ & 0.404 \\
\hline Axillary lymph node dissection & $81(29.2)$ & $23(27.1)$ & \\
\hline \multicolumn{4}{|l|}{ Charlson comorbidity index (CCI) } \\
\hline 0 & $69(24.9)$ & $10(11.8)$ & $<0.001$ \\
\hline 1 & $86(31.0)$ & $19(22.4)$ & \\
\hline 2 & $76(27.4)$ & $24(28.2)$ & \\
\hline $3+$ & $46(16.6)$ & $32(37.6)$ & \\
\hline \multicolumn{4}{|l|}{ Histologic type } \\
\hline Favorable (tubular, mucinous) & $29(10.5)$ & $10(11.8)$ & 0.435 \\
\hline Usual (IDC, ILC, Metaplastic, Mixed) & $248(89.5)$ & $75(88.2)$ & \\
\hline \multicolumn{4}{|l|}{ Histologic grade } \\
\hline 1 & $74(26.7)$ & $18(21.2)$ & 0.298 \\
\hline 2 & $129(46.6)$ & $41(48.2)$ & \\
\hline 3 & $74(26.7)$ & $26(23.5)$ & \\
\hline \multicolumn{4}{|l|}{ Stage } \\
\hline I & $147(53.1)$ & $38(44.7)$ & 0.270 \\
\hline$\|$ & $100(36.1)$ & $37(43.5)$ & \\
\hline III & $30(10.8)$ & $10(11.8)$ & \\
\hline \multicolumn{4}{|l|}{ T stage } \\
\hline $\mathrm{T} 1$ & $183(66.1)$ & $46(54.1)$ & 0.045 \\
\hline $\mathrm{T} 2$ & $86(31.0)$ & $35(41.2)$ & \\
\hline $\mathrm{T} 3$ & $8(2.9)$ & $4(4.7)$ & \\
\hline \multicolumn{4}{|l|}{ N stage } \\
\hline No & $200(72.2)$ & $60(70.6)$ & 0.702 \\
\hline N1 & $52(18.8)$ & $17(20.0)$ & \\
\hline N2 & $22(7.9)$ & $6(7.1)$ & \\
\hline N3 & $3(1.1)$ & $2(2.4)$ & \\
\hline \multicolumn{4}{|l|}{ Subtype } \\
\hline Luminal A & $156(56.3)$ & $46(54.1)$ & 0.563 \\
\hline Luminal B & $58(20.9)$ & $16(18.8)$ & \\
\hline HER2(+) & $25(9.0)$ & $6(7.1)$ & \\
\hline TNBC & $38(13.7)$ & $17(20.0)$ & \\
\hline \multicolumn{4}{|l|}{ Chemotherapy } \\
\hline Yes & $126(45.5)$ & $11(12.9)$ & $<0.001$ \\
\hline No & $151(54.5)$ & $74(87.1)$ & \\
\hline \multicolumn{4}{|l|}{ Radiotherapy } \\
\hline Yes & $177(63.9)$ & $37(43.5)$ & $<0.001$ \\
\hline No & $100(36.1)$ & $48(56.5)$ & \\
\hline \multicolumn{4}{|l|}{ Endocrine Therapy } \\
\hline Yes & $203(73.3)$ & $47(55.3)$ & 0.002 \\
\hline No & $74(26.7)$ & $38(44.7)$ & \\
\hline \multicolumn{4}{|l|}{ Trastuzumab } \\
\hline Yes & $27(9.7)$ & $6(7.1)$ & 0.304 \\
\hline No & $250(90.3)$ & $79(92.9)$ & \\
\hline \multicolumn{4}{|l|}{ Event } \\
\hline Distant metastasis & $14(5.1)$ & $8(9.4)$ & 0.115 \\
\hline Death & $24(8.7)$ & $13(15.3)$ & 0.063 \\
\hline
\end{tabular}

IDC = invasive ductal carcinoma; ILC = invasive lobular carcinoma; HER2 = human epidermal growth factor-2; TNBC = triple negative breast cancer. ${ }^{*} p$-values were calculated using the Chi-square test or Fisher's exact test. 
Table 2. Administration of adjuvant treatments by stage

\begin{tabular}{|c|c|c|c|c|c|c|c|}
\hline \multirow{2}{*}{ Stage } & & & \multicolumn{2}{|c|}{ Early-aged group } & \multicolumn{2}{|c|}{ Late-aged group } & \multirow{2}{*}{$p$-value } \\
\hline & & & No. & (\%) & No. & $(\%)$ & \\
\hline \multirow{6}{*}{ Stage I } & $\mathrm{CT}$ & Yes & 39 & $(26.5)$ & 3 & (7.9) & $<0.001$ \\
\hline & & No & 108 & (73.5) & 35 & $(92.1)$ & \\
\hline & ET & Yes & 109 & $(74.1)$ & 16 & $(42.1)$ & 0.024 \\
\hline & & No & 38 & $(25.9)$ & 22 & $(57.9)$ & \\
\hline & $\mathrm{RT}$ & Yes & 92 & $(62.6)$ & 24 & $(63.2)$ & 0.359 \\
\hline & & No & 55 & (37.4) & 14 & $(36.8)$ & \\
\hline \multirow[t]{6}{*}{ Stage II } & $\mathrm{CT}$ & Yes & 66 & $(66.0)$ & 6 & $(16.2)$ & $<0.001$ \\
\hline & & No & 34 & $(34.0)$ & 31 & $(83.8)$ & \\
\hline & ET & Yes & 73 & $(73.0)$ & 25 & $(67.6)$ & 0.244 \\
\hline & & No & 27 & $(27.0)$ & 12 & $(32.4)$ & \\
\hline & $\mathrm{RT}$ & Yes & 60 & $(60.0)$ & 16 & $(43.2)$ & 0.537 \\
\hline & & No & 40 & $(40.0)$ & 21 & $(56.8)$ & \\
\hline \multirow[t]{6}{*}{ Stage III } & $\mathrm{CT}$ & Yes & 21 & $(70.0)$ & 2 & $(20.0)$ & 0.005 \\
\hline & & No & 9 & $(30.0)$ & 8 & $(80.0)$ & \\
\hline & ET & Yes & 21 & $(70.0)$ & 6 & $(60.0)$ & 0.341 \\
\hline & & No & 9 & $(30.0)$ & 4 & $(40.0)$ & \\
\hline & $\mathrm{RT}$ & Yes & 25 & $(83.3)$ & 5 & $(50.0)$ & 0.089 \\
\hline & & No & 5 & $(16.7)$ & 5 & $(50.0)$ & \\
\hline
\end{tabular}

$\mathrm{CT}=$ chemotherapy; $\mathrm{ET}=$ endocrine therapy; $\mathrm{RT}=$ radiotherapy.

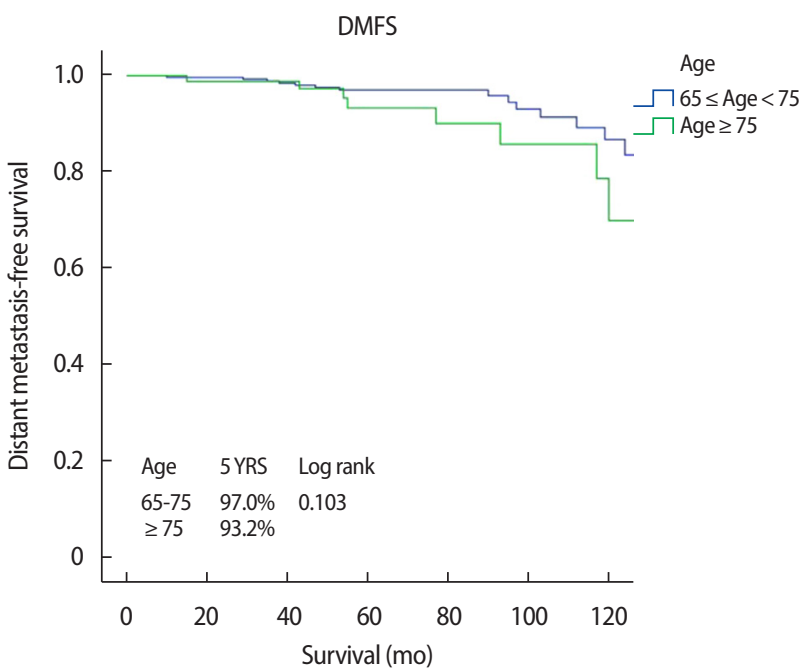

Figure 2. Distant metastasis-free survival and overall survival by age (A).

in patients with TNBC. Additionally, the late-aged group had poorer OS than that in the early-aged group $(91.8 \%$ vs. $94.0 \%, p=0.046)$. Figures 3 and 4 show DMFS and OS, respectively, by administration of chemotherapy, endocrine therapy, and radiotherapy in the early-aged and late-aged groups. In the early-aged group, chemotherapy was associated with worse DMFS while endocrine therapy was related to better DMFS and OS. Patients in the late-aged group who received chemotherapy had worse DMFS and OS than those who did not

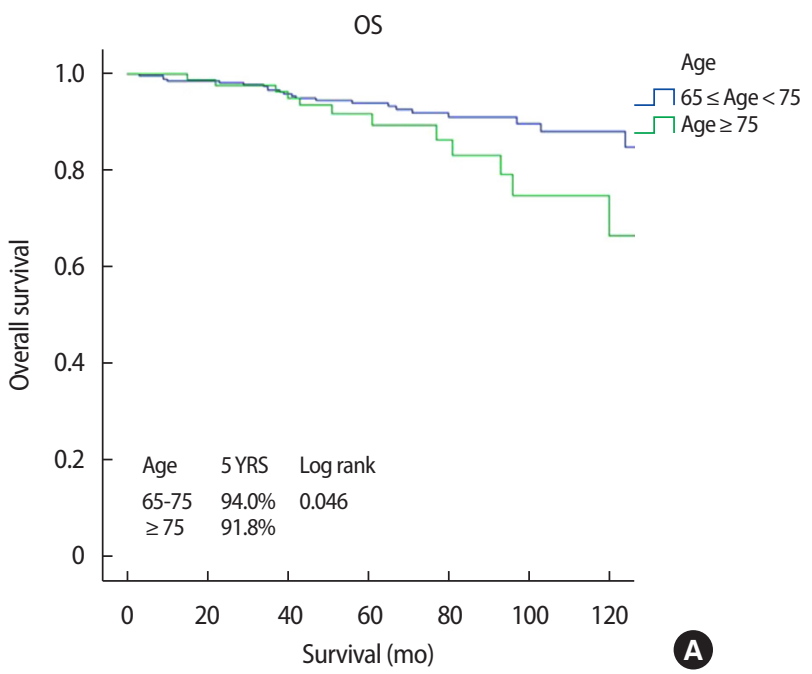

(Continued to the next page)

(DMFS, 63.6\% vs. 97.7\%, $p<0.001$; OS, 79.5\% vs. $93.6 \%, p=0.012$ ).

\section{Factors associated with survival}

The results of univariable and multivariable analyses to determine factors related to DMFS and OS are shown in Table 3. Multivariable analysis showed multiple comorbidities, high $\mathrm{HG}$, and advanced stage to be independent prognostic factors for poor DMFS and OS. However, subtype and administration of adjuvant treatments, including che- 

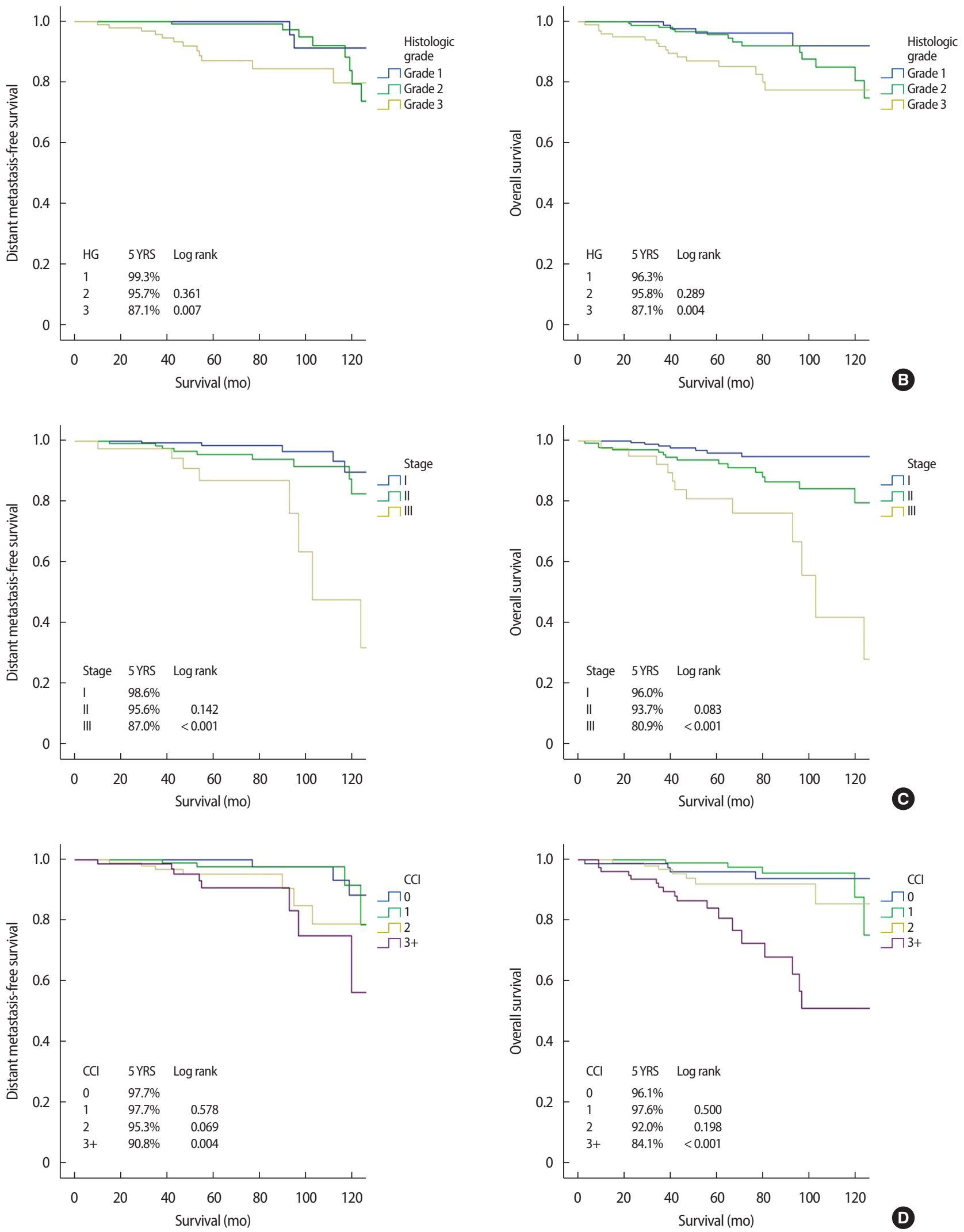

Figure 2. Continued. Histologic grade (B), stage (C), Charlson comorbidity index (D).

(Continued to the next page) 

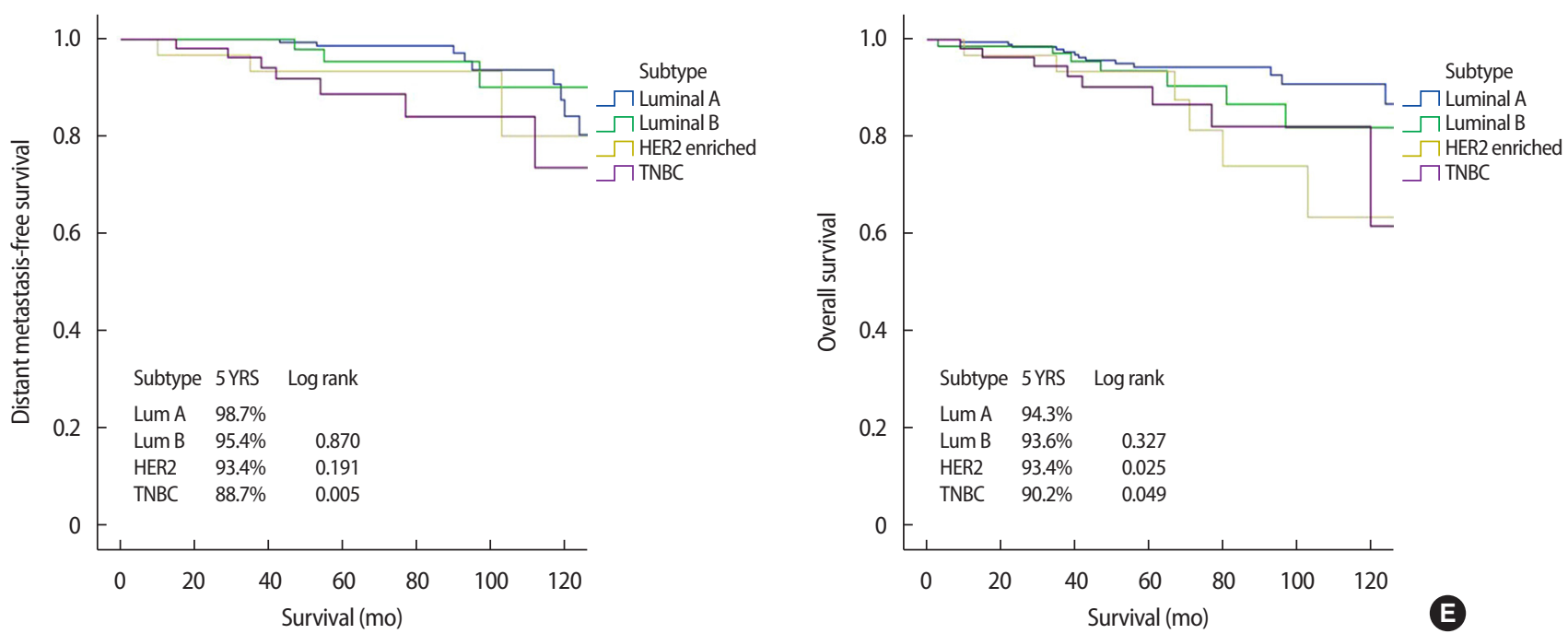

Figure 2. Continued. Subtype (E).

Table 3. Univariable and multivariable analysis for distant metastasis-free survival and overall survival

\begin{tabular}{|c|c|c|c|c|c|c|c|c|c|c|c|c|}
\hline \multirow{3}{*}{ Prognostic factor } & \multicolumn{6}{|c|}{ Distant metastasis-free survival } & \multicolumn{6}{|c|}{ Overall survival } \\
\hline & \multicolumn{3}{|c|}{ Univariate } & \multicolumn{3}{|c|}{ Multivariate } & \multicolumn{3}{|c|}{ Univariate } & \multicolumn{3}{|c|}{ Multivariate } \\
\hline & $p$-value & $\mathrm{HR}$ & $95 \% \mathrm{Cl}$ & $p$-value & $\mathrm{HR}$ & $95 \% \mathrm{Cl}$ & $p$-value & $\mathrm{HR}$ & $95 \% \mathrm{Cl}$ & $p$-value & $\mathrm{HR}$ & $95 \% \mathrm{Cl}$ \\
\hline \multicolumn{13}{|l|}{ Age group } \\
\hline $65 \leq$ Age $<75$ vs Age $\geq 75$ & 0.110 & 2.0 & $0.85-4.84$ & 0.519 & 1.5 & $0.45-4.89$ & 0.050 & 1.9 & $1.00-3.88$ & 0.823 & 0.9 & $0.39-2.09$ \\
\hline \multicolumn{13}{|l|}{$\mathrm{CCl}$} \\
\hline 0 & \multicolumn{3}{|c|}{ Ref. } & \multicolumn{3}{|c|}{ Ref. } & \multicolumn{3}{|c|}{ Ref. } & \multicolumn{3}{|c|}{ Ref. } \\
\hline 1 & 0.730 & 1.3 & $0.29-5.85$ & 0.795 & 1.2 & $0.27-5.54$ & 0.617 & 1.3 & $0.38-4.93$ & 0.663 & 1.3 & $0.37-4.75$ \\
\hline 2 & 0.071 & 3.5 & $0.89-13.7$ & 0.094 & 3.2 & $0.82-12.6$ & 0.125 & 2.5 & $0.76-8.58$ & 0.156 & 2.4 & $0.71-8.05$ \\
\hline 3 & 0.013 & 5.5 & $1.43-21.1$ & 0.021 & 4.9 & $1.27-19.2$ & $<0.001$ & 8.2 & $2.76-24.6$ & $<0.001$ & 7.6 & $2.54-23.01$ \\
\hline \multicolumn{13}{|l|}{ Histology grade } \\
\hline 1 & \multicolumn{3}{|c|}{ Ref. } & \multicolumn{3}{|c|}{ Ref. } & \multicolumn{3}{|c|}{ Ref. } & \multicolumn{3}{|c|}{ Ref. } \\
\hline 2 & 0.320 & 2.1 & $0.46-10.3$ & 0.575 & 1.5 & $0.32-7.59$ & 0.284 & 1.7 & $0.63-4.78$ & 0.541 & 1.4 & $0.49-3.84$ \\
\hline 3 & 0.024 & 5.6 & $1.25-25.2$ & 0.036 & 5.1 & $1.11-23.1$ & 0.011 & 3.6 & $1.34-9.92$ & 0.029 & 3.1 & $1.12-8.44$ \\
\hline \multicolumn{13}{|l|}{ Stage } \\
\hline I & \multicolumn{3}{|c|}{ Ref. } & \multicolumn{3}{|c|}{ Ref. } & \multicolumn{3}{|c|}{ Ref. } & \multicolumn{3}{|c|}{ Ref. } \\
\hline$\|$ & 0.143 & 2.2 & $0.75-6.77$ & 0.243 & 1.9 & $0.63-5.92$ & 0.087 & 2.0 & $0.90-4.50$ & 0.130 & 1.9 & $0.82-4.30$ \\
\hline III & $<0.001$ & 9.3 & $3.04-28.6$ & $<0.001$ & 9.2 & $2.92-29.3$ & $<0.001$ & 6.9 & $2.98-16.3$ & $<0.001$ & 6.4 & $2.68-15.2$ \\
\hline \multicolumn{13}{|l|}{ Subtype } \\
\hline Luminal A & \multicolumn{3}{|c|}{ Ref. } & \multicolumn{3}{|c|}{ Ref. } & \multicolumn{3}{|c|}{ Ref. } & \multicolumn{3}{|c|}{ Ref. } \\
\hline Luminal B & 0.923 & 1.1 & $0.28-3.94$ & 0.442 & 0.6 & $0.13-2.43$ & 0.380 & 1.4 & $0.61-3.65$ & 0.839 & 0.9 & $0.32-2.52$ \\
\hline HER2(+) & 0.218 & 2.2 & $0.61-8.41$ & 0.701 & 1.8 & $0.09-35.5$ & 0.038 & 2.7 & $1.05-7.02$ & 0.997 & 1.0 & $0.17-5.78$ \\
\hline TNBC & 0.011 & 3.6 & $1.34-9.75$ & 0.219 & 5.6 & $0.35-88.2$ & 0.069 & 2.2 & $0.93-5.16$ & 0.860 & 1.2 & $0.22-6.06$ \\
\hline Chemotherapy & & & & & & & & & & & & \\
\hline No vs Yes & 0.019 & 2.8 & $1.19-6.76$ & 0.122 & 2.6 & $0.77-8.64$ & 0.454 & 1.2 & $0.66-2.46$ & 0.649 & 0.8 & $0.34-1.93$ \\
\hline Radiotherapy & & & & & & & & & & & & \\
\hline No vs Yes & 0.706 & 1.1 & $0.50-2.76$ & 0.817 & 0.9 & $0.34-2.35$ & 0.165 & 0.6 & $0.31-1.21$ & 0.288 & 0.7 & $0.31-1.40$ \\
\hline Endocrine therapy & & & & & & & & & & & & \\
\hline No vs Yes & 0.063 & 0.4 & $0.19-1.04$ & 0.440 & 2.8 & $0.20-40.2$ & 0.040 & 0.5 & $0.26-0.96$ & 0.801 & 0.8 & $0.20-3.39$ \\
\hline
\end{tabular}

$\mathrm{HR}=$ hazard ratio; $\mathrm{Cl}=$ confidence interval; $\mathrm{CCl}=$ Charlson comorbidity index; $\mathrm{HER} 2=$ human epidermal growth factor receptor 2: TNBC= triple negative breast cancer. 

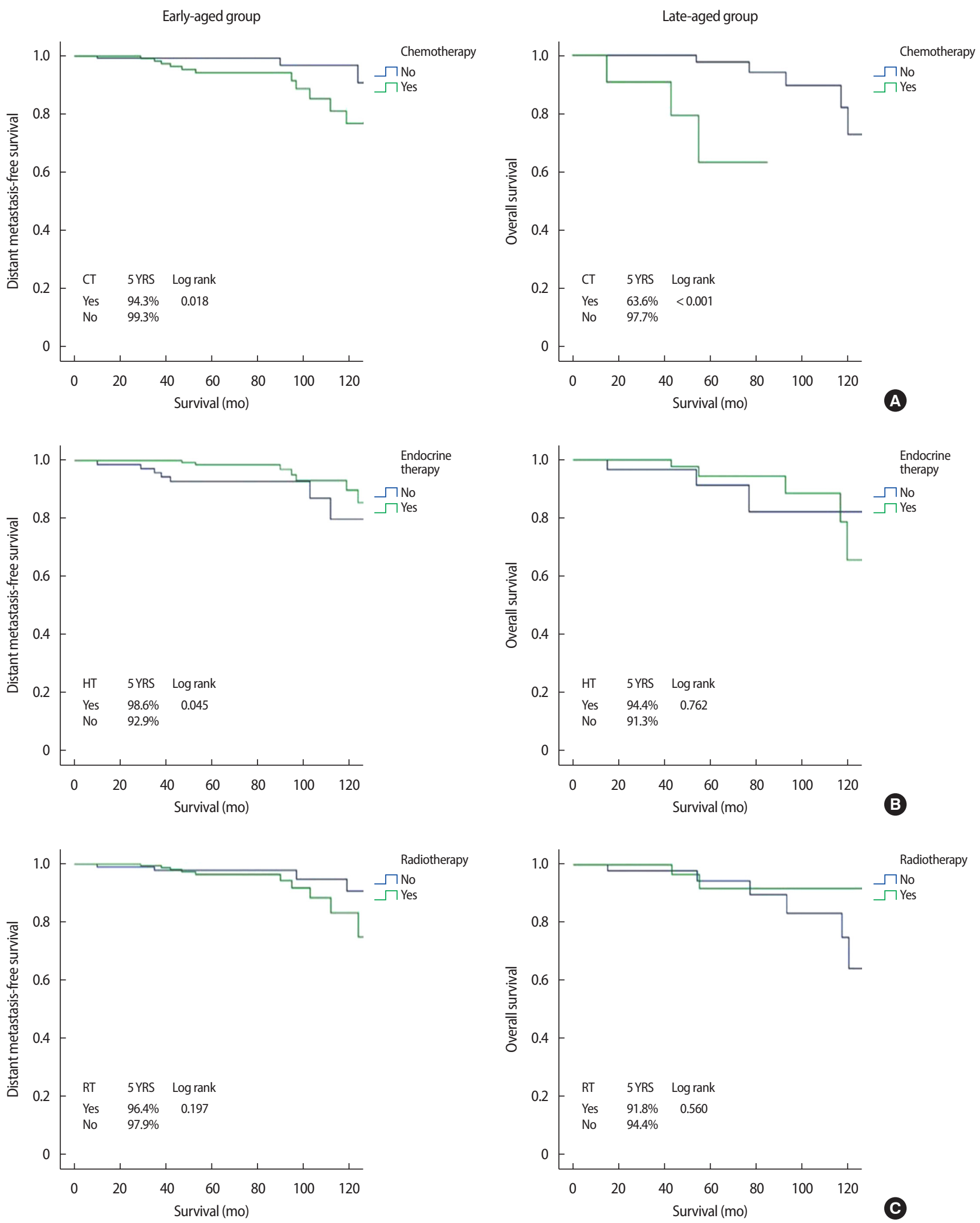

Figure 3. Distant metastasis-free survival according to adjuvant treatments between the early-aged and late-aged groups. 
Early-aged group
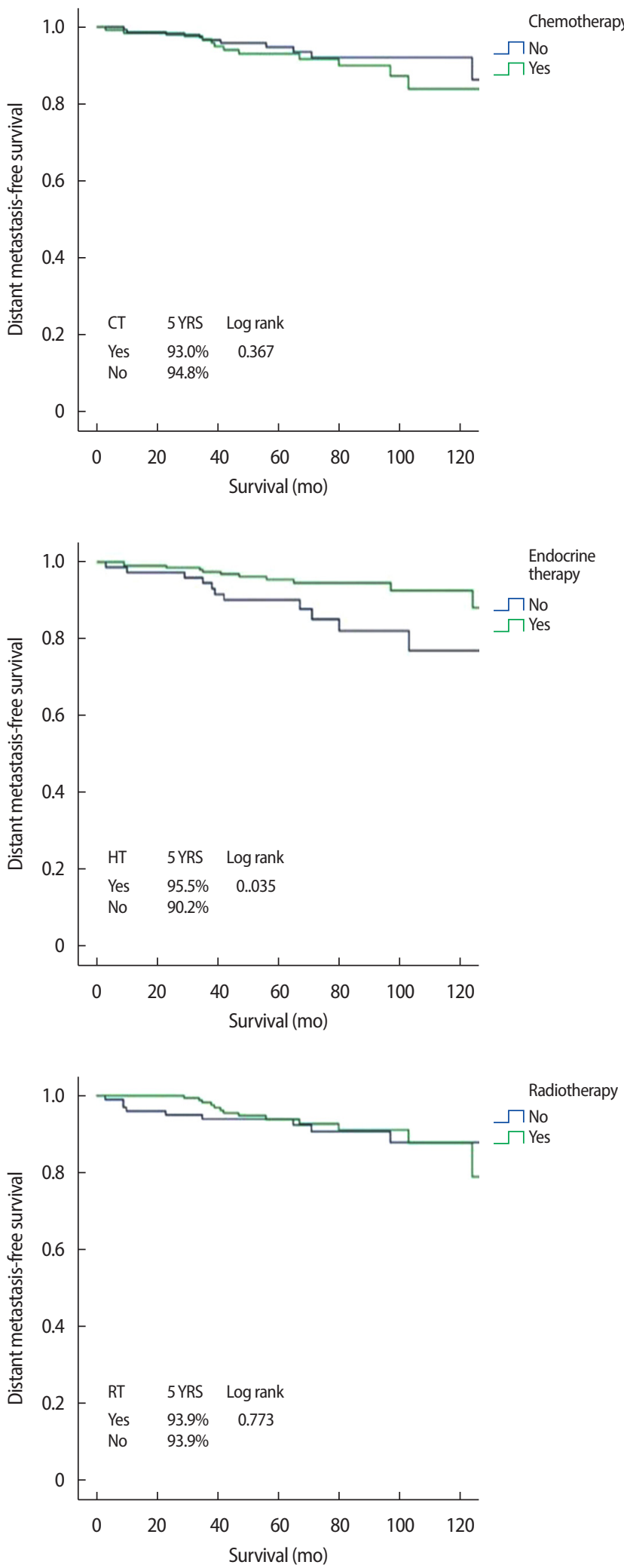

Late-aged group
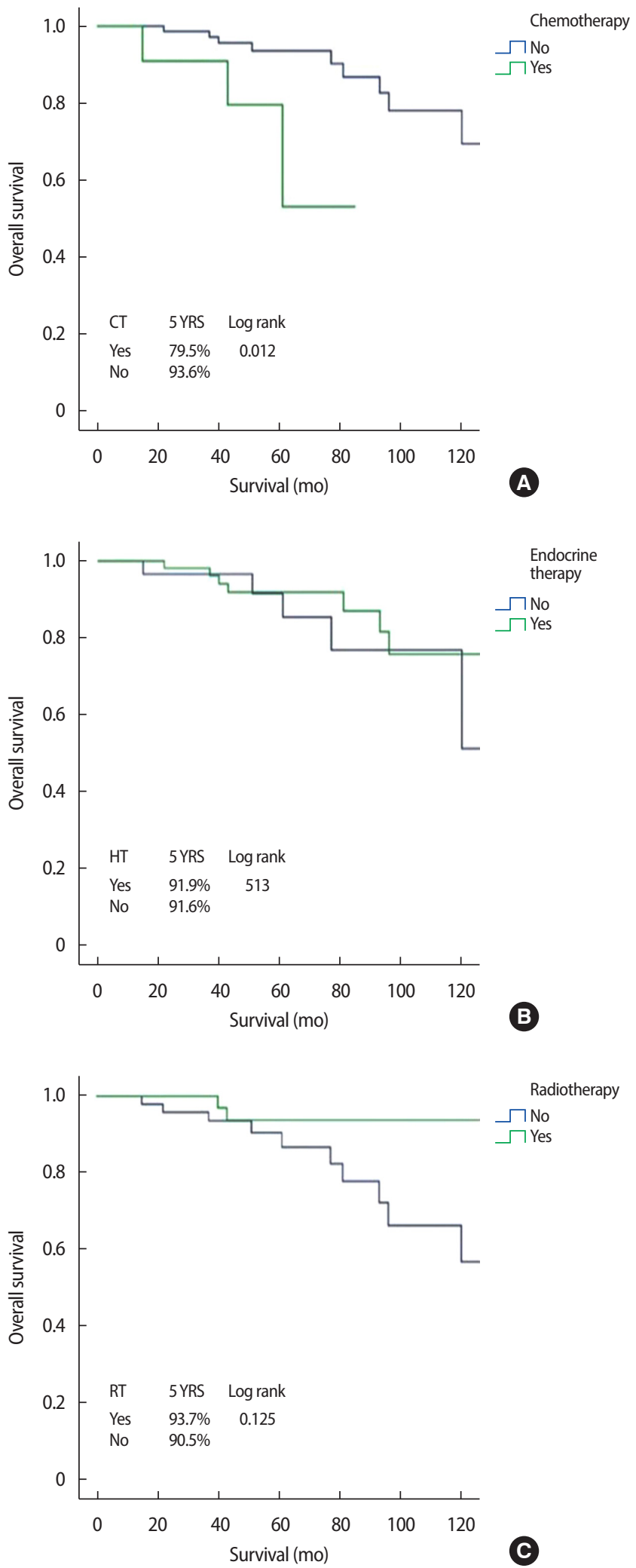

Figure 4. Overall survival according to adjuvant treatments between the early-aged and late-aged groups. 
motherapy, radiotherapy, and hormonal therapy, were not significantly associated with improved DMFS and OS.

\section{DISCUSSION}

This study evaluated the treatment patterns and clinical outcomes of elderly patients with breast cancer. Patients in the late-aged group ( $\geq 75$ years) had more comorbidities and were less likely to receive adjuvant chemotherapy and radiotherapy after breast cancer surgery. In multivariable analyses, higher comorbidities, HG, and stage were poor prognostic factors. The administration of adjuvant treatments did not affect prognosis in our elderly patients with breast cancer.

Several studies have reported that elderly breast cancer patients are less likely to receive adjuvant systemic therapy [19,20]. Hurria et al. [19] reported that only $5.2 \%$ of patients older than 75 years received adjuvant chemotherapy and that the proportion of patients receiving tamoxifen decreased with age (89\% of patients $75-79$ years and $79 \%$ of patients $\geq 80$ years). Yamada et al. [20] showed that patients were less likely to receive adjuvant systemic therapy as age increased $(93.6 \%$ of patients $\leq 65$ years, $90.5 \%$ of patients $65-74$ years, and $78.0 \%$ of patients $\geq 75$ years). Similarly, patients in the late-aged group in our study were also less likely to receive adjuvant systemic treatment even though similar oncological features were observed between the early-aged and late-aged groups. However, the International Society of Geriatric Oncology recommends that patients over 70 years of age receive the same treatment as younger patients in terms of surgery and adjuvant systemic therapy. Moreover, adjuvant chemotherapy should not simply be determined by age but should rather consider the patient's life expectancy and the potential benefit of treatment [21]. Bonadonna et al. [22] evaluated differences in recurrence and mortality among 386 patients over 75 years of age who underwent radical mastectomy $(\mathrm{n}=179)$ only or who also underwent 12 months of CMF after surgery $(n=207)$. They reported that adjuvant chemotherapy reduced the relative risk of relapse by $34 \%$ and the risk of mortality by $26 \%$ over 20 years. However, they did not consider the impact of comorbidities on prognosis. Some studies that have considered the effects of accompanying diseases have shown different clinical outcomes in elderly patients [23,24]. Braithwaite et al. [23] evaluated mortality according to CCI score $(0,1$, and $\geq 2)$ in 2272 patients, reporting that a higher CCI score was independently associated with an in- creased overall risk of death (hazard ratio [HR], 1.32; 95\% confidence interval [CI], 1.13-1.54), death from non-breast cancer causes (HR, 1.55; 95\% CI, 1.19-2.02), and breast cancer-specific death (HR, 1.47; 95\% CI, 1.03-2.09). Muss et al. [24] examined the toxic effects of adjuvant chemotherapy in three age groups ( $\leq 50$ years [ $n=3,506], 51-64$ years $[n=2,439]$, and $\geq 65$ years $[n=542]$ ), reporting higher chemotherapy-related mortality in elderly patients that increased linearly with age $(0.2 \%$ [ $\leq 50$ years], $0.7 \%$ [ $51-64$ years], and $1.5 \%$ [ $\geq 65$ years], $p<0.001)$. Multivariable analyses showed that smaller tumor size, fewer positive lymph nodes, less chemotherapy, and fewer comorbidities were significantly related to longer disease-free survival and OS $(p<0.001)$. Our study also showed a significant association between having more comorbidities (higher CCI score) and poor DMFS and OS in multivariable analysis. Moreover, the administration of adjuvant treatments, including chemotherapy, endocrine therapy, and radiotherapy, was not associated with improved DMFS and OS. These results may suggest that systemic treatment in elderly patients has the potential to worsen, rather than improve, health status and underlying diseases such as heart disease. Doyle et al. [25] reported cardiac toxicity associated with chemotherapy in elderly patients ( $>65$ years). The HRs of cardiomyopathy, congestive heart failure, and heart disease in patients treated with chemotherapy compared to those in patients who received no chemotherapy were 2.48 (95\% CI, 2.10-2.93), 1.38 (95\% CI, 1.25-1.52), and 1.35 (95\% CI, 1.26-1.44), respectively. Garg et al. [26] assessed the relationship between CCI and chemotherapy toxicity in 65 elderly ( $\geq 70$ years) patients and found increasing age, lower functional status, and high CCI to be associated with an increased discontinuation rate, dose reduction, and grade 3-4 toxicity.

Elderly patients with breast cancer are more likely to have ER-positive tumors that respond well to hormonal agents and are associated with a slightly better overall prognosis and reduced recurrence rate [27]. In the Early Breast Cancer Trialists' Collaborative Group analysis of patients with ER-positive breast cancer, 5 years of adjuvant endocrine therapy with tamoxifen reduced the annual recurrence rate at 15 years by $34 \%$ in women aged $50-59,45 \%$ in women aged $60-69$, and $51 \%$ in women older than 70 years. Among these patient groups, the annual death rate was also reduced by $24 \%, 35 \%$, and $37 \%$, respectively [28]. In another study, the effects of adjuvant endocrine therapy differed based on comorbidity. Land et al. [29] evaluated the breast cancer-specific survival (BCSS) by CCI score in elderly patients who re- 
ceived endocrine therapy, reporting lower BCSS in patients with higher CCI scores $(1,2$, and $\geq 3)$ than that in patients without comorbidity (HR 1.23 [95\% CI, 1.09-1.39] for CCI 1, HR 1.28 [95\% CI, 1.051.55] for CCI 2, and HR 1.60 [95\% CI, 1.23-2.07] for CCI 3). We also observed an increased risk of systemic relapse and overall mortality with increasing CCI score, but they were not affected by endocrine therapy.

Molecular subtype is a well-known prognostic factor of breast cancer, with the TNBC and luminal A subtypes showing the worst and the best prognoses, respectively. However, some studies have reported no difference in prognosis according to subtype in elderly patients. de Kruijf et al. [30] reported an association between molecular subtypes and prognosis in young patients ( $<65$ years; $n=361$ ) based on the relapse-free period (RFP) $(p=0.01)$ and relative survival (RS) $(p<0.001)$. However, they observed no significant prognostic effect of subtype in elderly patients ( $\geq 65$ years; $\mathrm{n}=189$; RFP $p=0.5$; RS $p=0.1$ ). Similarly, multivariable analysis in the present study also revealed no significant difference in prognosis based on subtype.

This study has several limitations. First, our study was a retrospective study performed in a single institution. A critical drawback of the retrospective design was that the therapeutic decision-making process in elderly patients was not clear. It was difficult to prove a causal relationship between adjuvant treatment and survival improvement. In elderly patients, there is the potential for selection bias because adjuvant therapies may be limited depending on accompanying disease, age, or patient preferences. Moreover, the small number of samples made it difficult to perform meaningful multivariable analysis of heterogeneous factors associated with treatment. Second, we could not analyze BCSS. The cause of death for patients who continued to visit our hospital was confirmed by EMR. However, due to follow-up loss, the cause of death could not be identified in $43 \%$ of all deaths. We, therefore, evaluated the overall mortality and related prognostic factors. Third, CCI is generally a tool used to assess comorbidity but does not reflect disease severity or patient performance status. A more comprehensive assessment of comorbidity in elderly patients, such as the Comprehensive Geriatric Assessment, could assist in determining the treatment process.

Recent demographic changes suggest that the number of older patients with breast cancer will continue to increase; thus, clinicians need to be prepared to treat this patient population. Although it is rec- ommended that elderly breast cancer patients be as actively treated as young patients, the results of our study showed that adjuvant treatments were not associated with prognosis; furthermore, higher $\mathrm{HG}$, stage, and CCI score were independently associated with poor prognosis in multivariate analysis. Therefore, treatment planning for elderly patients must consider various factors including not only important clinical features such as stage and subtype but also comorbidities, performance status, and life expectancy. Since, as a retrospective study in a single institution, this study has several limitations, it is difficult to conclude the effect of adjuvant therapy in elderly patients. Guidelines must be established for the treatment of breast cancer in elderly patients and the study of its therapeutic effects should be expanded.

\section{CONFLICT OF INTEREST}

The authors declare that they have no competing interests.

\section{ACKNOWLEDGMENTS}

The authors thank the Division of Statistics of the Medical Research Collaborating Center at Seoul National University Bundang Hospital for performing the statistical analyses.

\section{REFERENCES}

1. Definition of an older or elderly person. WHO, Geneva: Switzerland; 2010. http://www.who.int/healthinfo/survey/ageingdefnolder/en/index.html. Accessed Nov 12th, 2018.

2. 2016 Statistical report on the aged. Korea National Statistical Office. http://kostat.go.kr/portal/korea/kor_nw. Accessed Nov 12th, 2018.

3. Kontis V, Bennett JE, Mathers CD, Li G, Foreman K, Ezzati M. Future life expectancy in 35 industrialised countries: projections with a bayesian model ensemble. Lancet 2017;389:1323-35

4. Karuturi M, VanderWalde N, Muss H. Approach and management of breast cancer in the elderly. Clin Geriatr Med 2016;32:133-53.

5. Berglund A, Wigertz A, Adolfsson J, Ahlgren J, Fornander T, Warnberg $\mathrm{F}$, et al. Impact of comorbidity on management and mortality in women diagnosed with breast cancer. Breast Cancer Res Treat 2012;135:281-9.

6. Wallwiener CW, Hartkopf AD, Grabe E, Wallwiener M, Taran FA, 
Fehm T, et al. Adjuvant chemotherapy in elderly patients with primary breast cancer: are women $\geq 65$ undertreated? J Cancer Res Clin Oncol 2016;142:1847-53.

7. Owusu C, Lash TL, Silliman RA. Effect of undertreatment on the disparity in age-related breast cancer-specific survival among older women. Breast Cancer Res Treat 2007;102:227-36.

8. Muss HB, Berry DA, Cirrincione CT, Theodoulou M, Mauer AM, Kornblith AB, et al. Adjuvant chemotherapy in older women with early-stage breast cancer. N Engl J Med 2009;360:2055-65.

9. Bouchardy C, Rapiti E, Fioretta G, Laissue P, Neyroud-Caspar I, Schafer P, et al. Undertreatment strongly decreases prognosis of breast cancer in elderly women. J Clin Oncol 2003;21:3580-7.

10. Dehal A, Abbas A, Johna S. Comorbidity and outcomes after surgery among women with breast cancer: analysis of nationwide in-patient sample database. Breast Cancer Res Treat 2013;139:46976.

11. Houterman S, Janssen-Heijnen ML, Verheij CD, Louwman WJ, Vreugdenhil G, van der Sangen MJ, et al. Comorbidity has negligible impact on treatment and complications but influences survival in breast cancer patients. Br J Cancer 2004;90:2332-7.

12. Klepin HD, Pitcher BN, Ballman KV, Kornblith AB, Hurria A, Winer EP, et al. Comorbidity, chemotherapy toxicity, and outcomes among older women receiving adjuvant chemotherapy for breast cancer on a clinical trial: CALGB 49907 and CALGB 361004 (alliance). J Oncol Pract 2014;10:e285-92.

13. Wildes TM, Ruwe AP, Fournier C, Gao F, Carson KR, Piccirillo JF, et al. Geriatric assessment is associated with completion of chemotherapy, toxicity, and survival in older adults with cancer. J Geriatr Oncol 2013;4:227-34.

14. Yancik R, Wesley MN, Ries LA, Havlik RJ, Edwards BK, Yates JW. Effect of age and comorbidity in postmenopausal breast cancer patients aged 55 years and older. JAMA 2001;285:885-92.

15. Charlson ME, Pompei P, Ales KL, MacKenzie CR. A new method of classifying prognostic comorbidity in longitudinal studies: development and validation. J Chronic Dis 1987;40:373-83.

16. Edge SB, Compton CC. The American Joint Committee on Cancer: the 7th edition of the AJCC cancer staging manual and the future of TNM. Ann Surg Oncol 2010;17:1471-4.

17. Goldhirsch A, Winer EP, Coates AS, Gelber RD, Piccart-Gebhart M, Thurlimann B, et al. Personalizing the treatment of women with early breast cancer: highlights of the st gallen international expert consensus on the primary therapy of early breast cancer 2013. Ann Oncol 2013;24:2206-23.

18. Sesterhenn AM, Albert US, Barth PJ, Wagner U, Werner JA. The status of neck node metastases in breast cancer-loco-regional or distant? Breast 2006;15:181-6

19. Hurria A, Leung D, Trainor K, Borgen P, Norton L, Hudis C. Factors influencing treatment patterns of breast cancer patients age 75 and older. Crit Rev Oncol Hematol 2003;46:121-6.

20. Yamada A, Narui K, Sugae S, Shimizu D, Takabe K, Ichikawa Y, et al. Operation with less adjuvant therapy for elderly breast cancer. J Surg Res 2016;204:410-7.

21. Biganzoli L, Wildiers H, Oakman C, Marotti L, Loibl S, Kunkler I, et al. Management of elderly patients with breast cancer: updated recommendations of the International Society of Geriatric Oncology (SIOG) and European Society of Breast Cancer Specialists (EUSOMA). Lancet Oncol 2012;13:e148-60.

22. Bonadonna G, Valagussa P, Moliterni A, Zambetti M, Brambilla C. Adjuvant cyclophosphamide, methotrexate, and fluorouracil in node-positive breast cancer: the results of 20 years of follow-up. $\mathrm{N}$ Engl J Med 1995;332:901-6.

23. Braithwaite D, Moore DH, Satariano WA, Kwan ML, Hiatt RA, Kroenke C, et al. Prognostic impact of comorbidity among longterm breast cancer survivors: results from the LACE study. Cancer Epidemiol Biomarkers Prev 2012;21:1115-25.

24. Muss HB, Woolf S, Berry D, Cirrincione C, Weiss RB, Budman D, et al. Adjuvant chemotherapy in older and younger women with lymph node-positive breast cancer. JAMA 2005;293:1073-81.

25. Doyle JJ, Neugut AI, Jacobson JS, Grann VR, Hershman DL. Chemotherapy and cardiotoxicity in older breast cancer patients: a population-based study. J Clin Oncol 2005;23:8597-605.

26. Garg P, Rana F, Gupta R, Buzaianu EM, Guthrie TH. Predictors of toxicity and toxicity profile of adjuvant chemotherapy in elderly breast cancer patients. Breast J 2009; 15:404-8.

27. Lodi M, Scheer L, Reix N, Heitz D, Carin AJ, Thiebaut N, et al. Breast cancer in elderly women and altered clinico-pathological characteristics: a systematic review. Breast Cancer Res Treat 2017;166:657-68.

28. Early Breast Cancer Trialists' Collaborative G. Effects of chemotherapy and hormonal therapy for early breast cancer on recurrence 
and 15-year survival: an overview of the randomised trials. Lancet 2005;365:1687-717.

29. Land LH, Dalton SO, Jensen MB, Ewertz M. Influence of comorbidity on the effect of adjuvant treatment and age in patients with early-stage breast cancer. Br J Cancer 2012;107:1901-7.
30. de Kruijf EM, Bastiaannet E, Ruberta F, de Craen AJ, Kuppen PJ, Smit VT, et al. Comparison of frequencies and prognostic effect of molecular subtypes between young and elderly breast cancer patients. Mol Oncol 2014;8:1014-25. 